\title{
A Generic Linked List Implementation in Fortran $95^{*}$
}

\author{
JASON R. BLEVINS ${ }^{+}$ \\ Department of Economics, Duke University
}

May 18, 2009

\begin{abstract}
This paper develops a standard conforming generic linked list in Fortran 95 which is capable of storing data of an any type. The list is implemented using the transfer intrinsic function, and although the interface is generic, it remains relatively simple and minimizes the potential for error. Although linked lists are the focus of this paper, the generic programming techniques used are very general and broadly-applicable to other data structures and procedures implemented in Fortran 95 that need to be used with data of an unknown type.
\end{abstract}

Keywords: Fortran 95, generic programming, data structures, scientific computing.

\section{INTRODUCTION}

A linked list, or more specifically a singly-linked list, is a list consisting of a series of individual node elements where each node contains a data member and a pointer that points to the next node in the list. This paper describes a generic linked list implementation in standard Fortran 95 which is able to store arbitrary data, and in particular, pointers to arbitrary data types. While linked lists are the focus of this paper, the underlying idea can be easily used to create more general generic data structures and procedures in Fortran 95. The generic_list module is developed in detail below, followed by an example control program which illustrates the list interface, showing how to use Fortran's transfer intrinsic function to store and retrieve pointers to derived type data variables.

This module has several advantages: it is written in standard Fortran 95 to ensure portability, it is self-contained and does not require the use of a preprocessor or include statements, and the transfer function only needs to be called once by the user, resulting in clean code and minimizing the potential for errors. Furthermore, simple wrapper functions can be written for specific types so that transfer would not need to be called at all by the user.

*The author benefited greatly from Arjen Markus's open source FLIBS project at http://flibs. sourceforge. net/, which illustrates several generic programming techniques, and from reading Richard Maine's descriptions of using transfer to pass pointers, posted to comp. lang. fortran.

${ }^{\dagger}$ Email: jason.r.blevins@duke.edu 
Several other generic list implementations have been offered, each with its own advantages and disadvantages. The generic list of McGavin and Young (2001) is very similar to the one presented here, but it is not standard-conforming due to an assumption about the physical representation of pointers to derived types. The method described below avoids this problem by writing the list interface in such a way that the compiler automatically determines the exact amount of storage required for any arbitrary data type the user wishes to store in the list.

The FLIBS project (Markus, 2008) provides a generic linked list in the form of an include file. The user then creates a new module for each data type that will be stored in a list. The module then includes the generic list code. This approach is clean in that the compiler can perform type checking to reduce errors, however, it requires the user to create potentially many new modules and each list can only store data of a single type. The method described in this paper does not require the user to write any additional code.

The generic_list module it is not, however, without flaws. In particular, the transfer function can be confusing for new Fortran programmers and must be used with care to avoid subtle errors. Furthermore, a disadvantage with respect to type-aware lists is that when storing pointers to data elements in the generic list, the elements must be allocated and deallocated by the user. This is the case with all dynamically allocated memory in Fortran, but it is nonetheless an area where care should be taken when implementing the generic programming techniques used herein.

While the generic_list implementation in this paper uses the transfer function, other generic programming techniques are also possible. A preprocessor such as the $\mathrm{C}$ preprocessor, m4, or a Fortran-specific preprocessor such as Forpedo (McCormack, 2005) can be used to automatically generate specific code from generic code. Markus (2001) discusses generic programming in Fortran 90 using both text substitution and implementation-hiding. The former is a form of preprocessing while the latter is accomplished by writing, say, a quicksort (Hoare, 1961) routine in such a way that it requires passing only two procedures for swapping and comparing data elements, rather than passing the data array itself. The swap and compare procedures have a generic interface and so the resulting sort routine is type-independent.

It is also worth nothing that with the introduction of Fortran 2003, the methods discussed in this paper will no longer be necessary. Instead, new features such as unlimited polymorphic (class $(*))$ objects or C pointers (variables of type(c_ptr)) can be used to implement generic data structures in a more natural way. However, until Fortran 2003 compliant compilers become widely available, Fortran 95 techniques such as these will remain useful.

In the following, we first briefly review Fortran pointers and the transfer intrinsic before presenting the generic_list module and an example program. 


\section{POINTERS AND THE TRANSFER INTRINSIC}

Fortran 95 has no syntax for constructing arrays of pointers and it requires some additional work to manipulate pointers themselves rather than the pointer targets. A common idiom involves constructing a derived type containing only a pointer to the data in question. The same approach can be used to store pointers in the generic list presented here. Consider, for example, a representative data type called data_t and an associated derived type for storing data_t pointers called data_ptr.

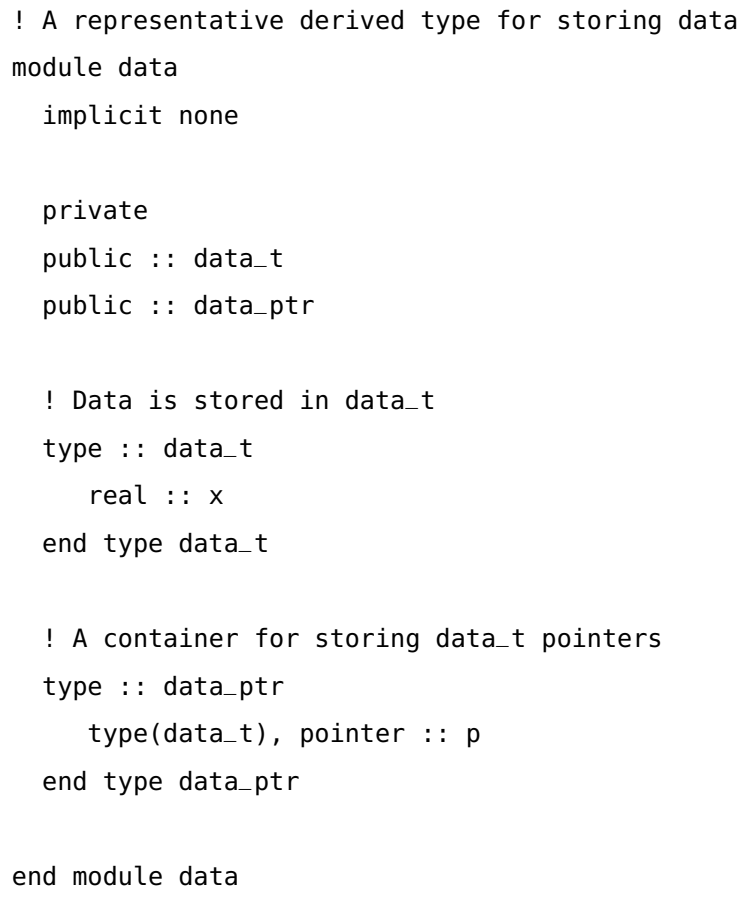

The data_t type is very simple, holding only a single real variable, but such data container types can easily become very complex in specific applications. As such, rather than using transfer to copy entire data_t structures in and out of the list, it is sometimes desirable to copy the smaller data_ptr structures instead. The data_ptr type also allows us to create arrays of pointers:

type(data_ptr), dimension(100) : : pointer_array

In most cases, it is these pointers that we would like to store in the linked list nodes.

Rather than build a new list for each possible type we might want to store, we use the transfer function to convert data of an arbitrary type to an array of integers before storing it in the list. We thus only have to write one linked-list module which is capable of handling rank-one integer arrays.

The transfer function introduced in Fortran 90 can be used to move data of one type through procedures and variables that were expecting data of some other type. In this 
sense, transfer can approximate the behavior of void pointers in C. Essentially, it copies the bits in memory representing a variable source to a scalar or array of the same type as a given mold. The syntax for transfer is

result $=\operatorname{transfer}($ source, $\operatorname{mold}[$, size $])$

where source and mold are scalars or arrays of any type and size is an optional scalar of type integer. The result is of the same type as mold. If size is given, result is a rank-one array of length size. If size is omitted but mold is an array, then result is an array just large enough to represent the source. Finally, if size is omitted and mold is a scalar, then result is a scalar.

Returning to our data_ptr pointer example above, we can convert pointers to integer arrays by using transfer as follows:

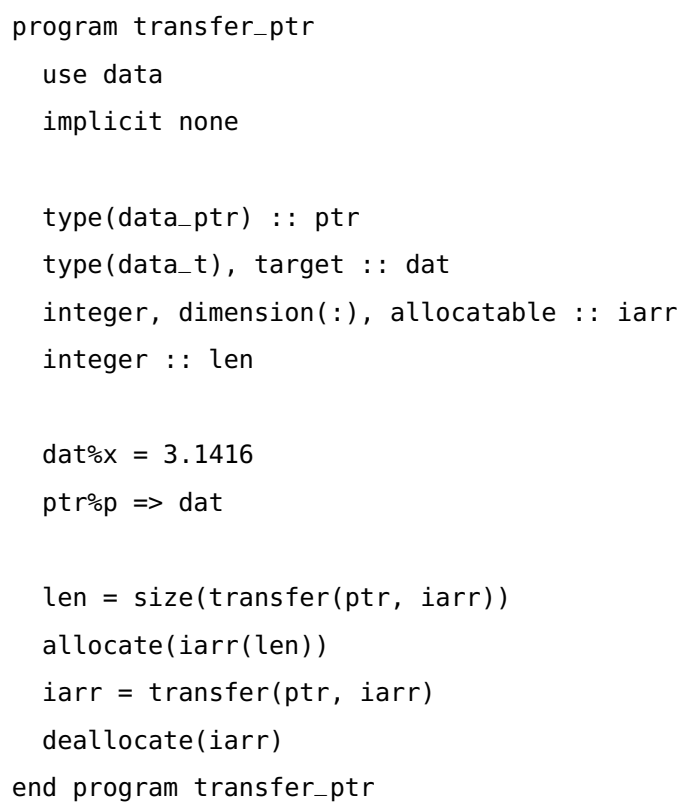

The above code makes two calls to transfer: first, to probe the size of the array needed to store the pointer in integer form, and second, to actually transfer the data. We must check the size for generality. It might be the case that the data_t pointer uses the same amount of storage as a single default integer, but the Fortran standard does not guarantee this. Furthermore, we might wish to store other types of data in the list, not just pointers.

On the author's system, for one run of the transfer-ptr program above, the values of len and iarr were 2 and $(1054311824,32767)$ respectively. Note that the value of iarr represents the location of dat in memory and will likely differ each time the program is executed.

The transfer construction above is verbose, error prone, and inefficient. The interface of the generic_list implementation is designed to minimize the inconvenience of using 
transfer so that only a single call to transfer is required to store or retrieve data from the list. Instead of manually allocating the temporary array, we can use the transfer statement as an argument expression, along with assumed-shape arrays in the list interface, and let the compiler determine the appropriate size, thus avoiding one call each to size and transfer. Although the compiler will create a temporary array, it replaces the manually-allocated array in the above example which will not be required.

\section{IMPLEMENTATION}

The generic_list module below defines the list_node_t type, from which the list is constructed, and the related procedures for initializing and freeing the list and manipulating list nodes. Although the data element of each list node is defined as a pointer to an array of integers, the list is not intended to simply store integers. Rather, Fortran's transfer intrinsic will be used to "encode" any arbitrary data type so that it can be represented as an array of integers. When data needs to be accessed later, transfer is used again to "decode" it, returning it to its original type. Using the methods of the previous section, we can also store pointers to arbitrary data types in the list.

The module first defines a few types including the list_node_t type which stores the data and well as a variable list_data which is defined for convenience to be used as a mold for transfer.

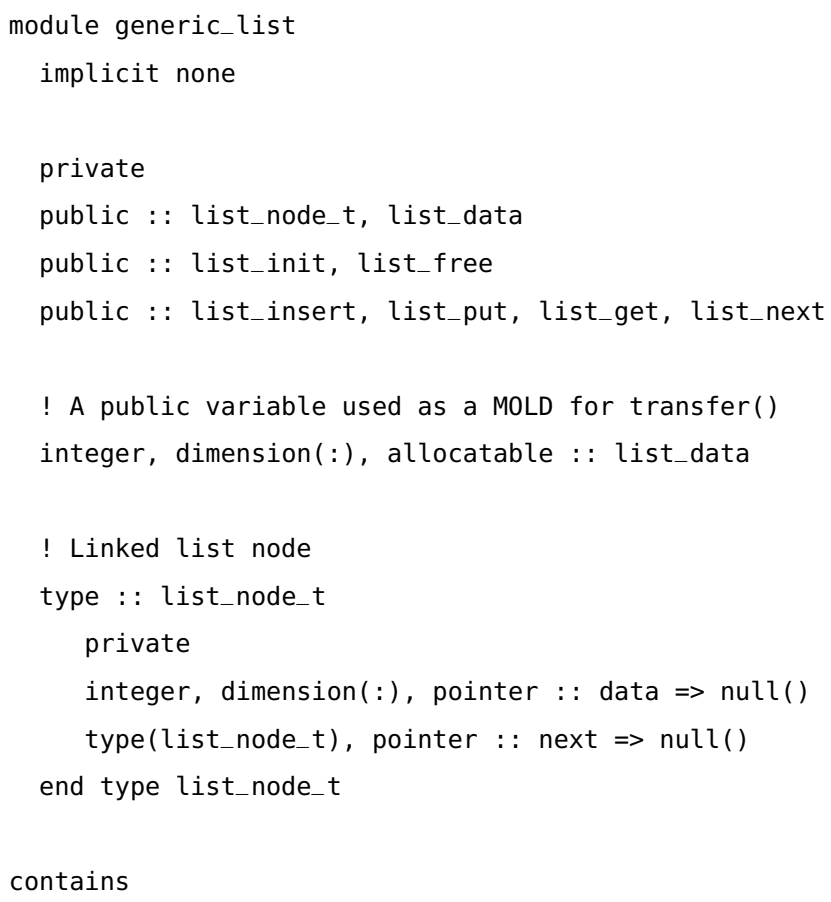




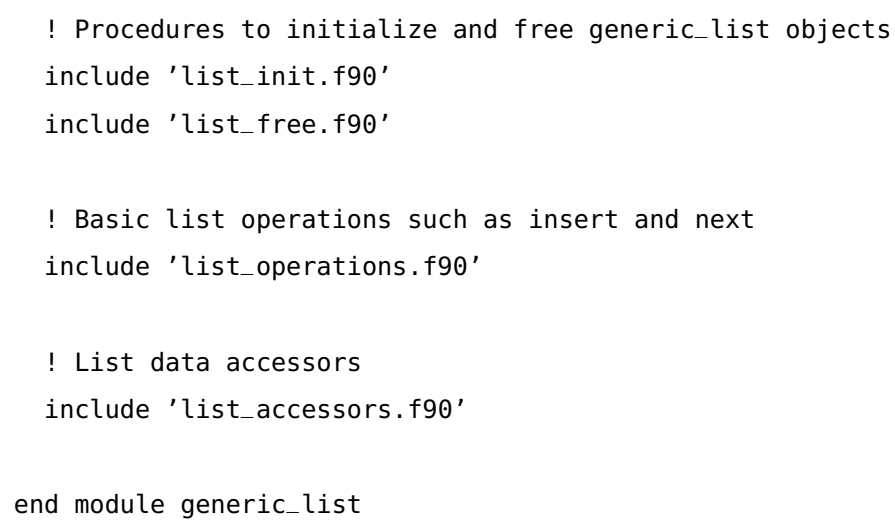

Two life-cycle procedures are defined to initialize memory for the list (list_init) and to free the memory once the list is no longer needed (list_free). The list_init subroutine allocates memory for a "head node" and nullifies the next pointer. It also takes an optional data argument to allow the first data object to be stored upon initialization.

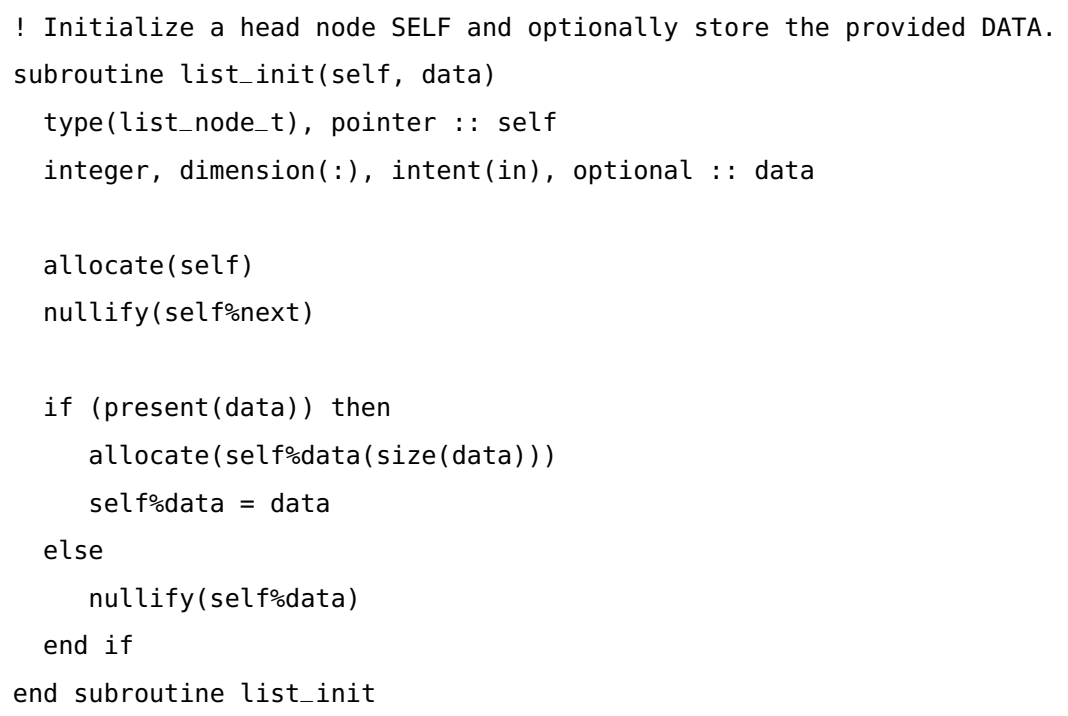

The list_free procedure traverses the list, deallocating list nodes and their data until the end of the list is reached, as indicated by a node with a null next pointer.

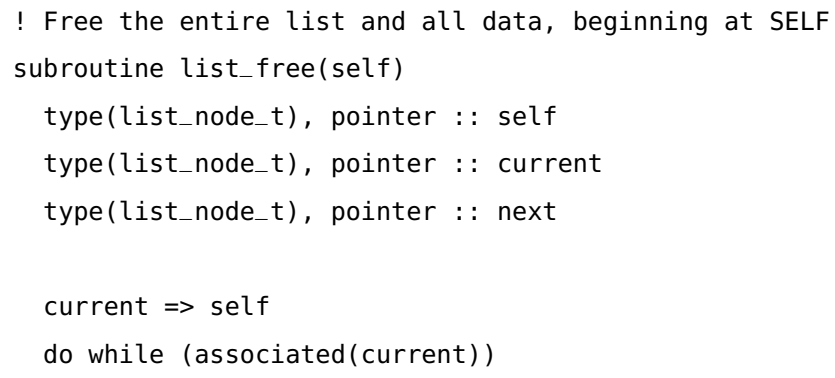




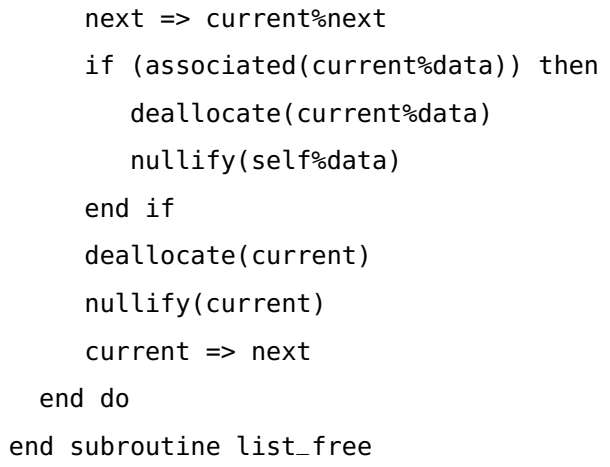

Since the list node data is encapsulated by the private attribute in order to hide the implementation, three simple accessor procedures list_put, list_get, and list_next are also defined. list_put stores encoded data in a particular list node and list_get retrieves data. The list_next function returns the next node in the list.

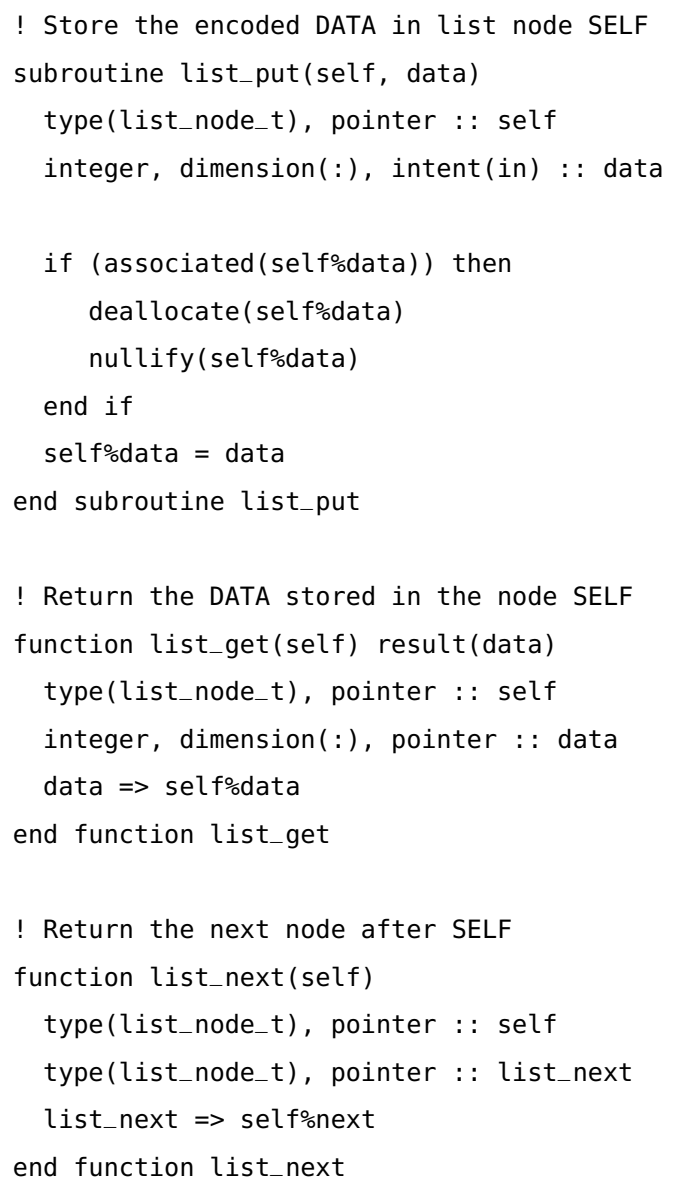

Although many additional list operations could be defined, we provide only a single 
example list_insert procedure which inserts a new node after the current one.

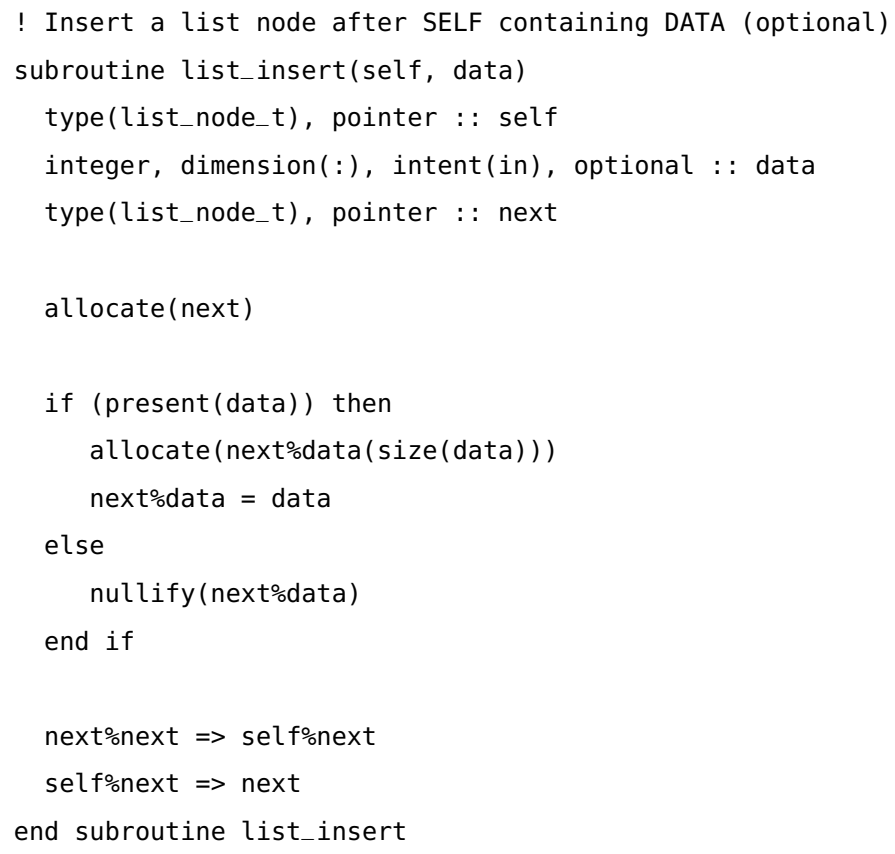

Finally, we provide a simple control program which illustrates how to initialize and free the list and how to store and retrieve pointers to data_t objects using transfer.

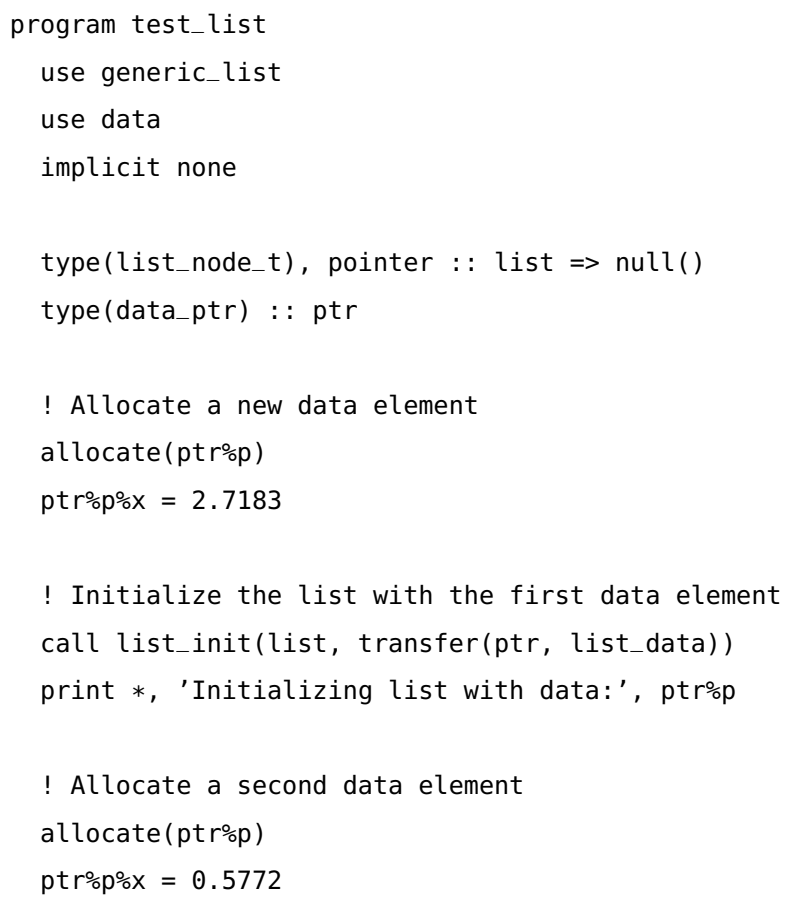




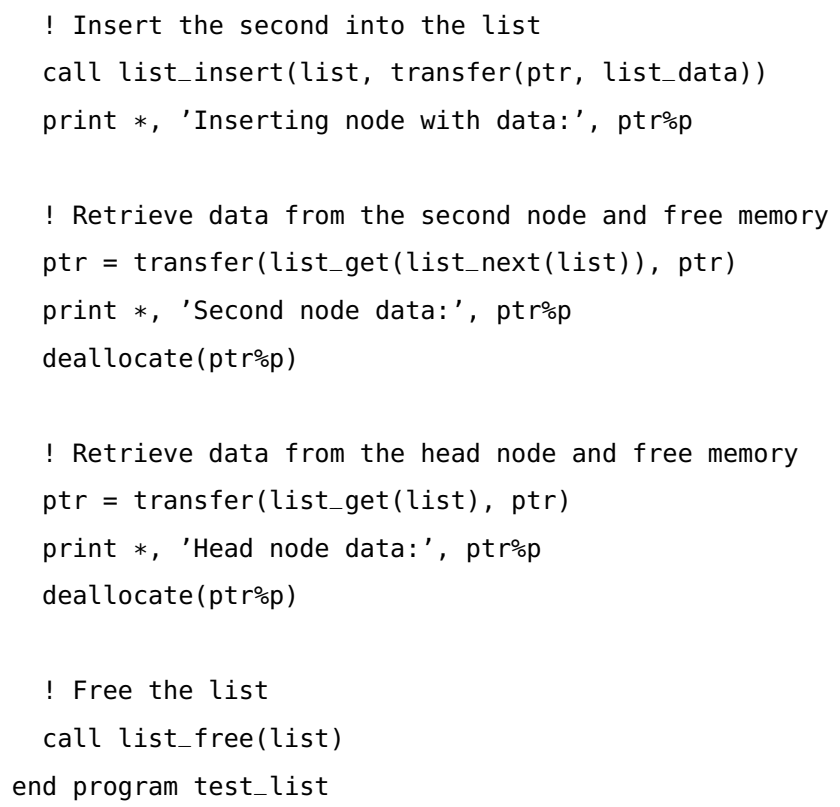

The test program produces the following output:

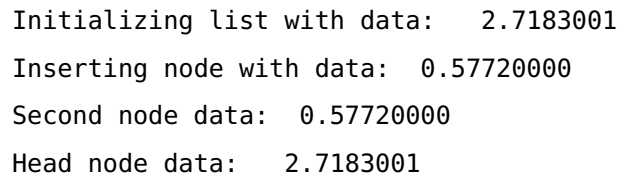

\section{CONCLUSION}

The generic programming methods described in this paper provide a relatively simple way to structure Fortran 95 modules and procedures that need to be used with data of an unknown type. These methods are general and broadly-applicable to data structures and procedures far beyond the simple linked list considered in this paper. In specific applications, a few simple wrapper functions can be written for the required data types so that the resulting interface is clean and simple, entirely avoiding direct use of transfer by the user. Until the new language features of Fortran 2003 become more accessible, generic Fortran 95 techniques such as these can provide much of the same convenience and functionality at the cost of a slightly more complicated user interface.

\section{REFERENCES}

Hoare, C. A. R. (1961). Quicksort: Algorithm 64. Communications of the ACM 4, 321-322. [2]

Markus, A. (2001). Generic programming in Fortran 90. ACM SIGPLAN Fortran Forum 20(3), 20-23. [2] 
Markus, A. (2008). FLIBS - a collection of Fortran modules. http://flibs . sourceforge . net/. Version o.9. [2]

McCormack, D. (2005). Generic programming in Fortran with Forpedo. ACM SIGPLAN Fortran Forum 24(2), 18-29. [2]

McGavin, P. and R. Young (2001). A generic list implementation. ACM SIGPLAN Fortran Forum 20(1), 16-20. [2] 